Tema: Aciaria Oxigênio

\title{
PARTIÇÃO DE FÓSFORO NOS CONVERTEDORES DA ARCELORMITTAL TUBARÃO*
}

\section{Resumo}

\author{
Walter Luiz Corrêa Junior ${ }^{1}$ \\ Henrique Silva Furtado ${ }^{2}$ \\ Raphael de Alcantara Sampaio ${ }^{3}$ \\ José Roberto de Oliveira ${ }^{4}$
}

O fósforo é um dos elementos mais importantes do processo de produção de aço em convertedores a oxigénio, devido à dificuldade de remoção durante o processo de sopro de oxigénio e depois do vazamento para panela de aço. Este trabalho mostra resultados obtidos através da alteração em variáveis de processo como o consumo de cales, teor de FeO na escória e uso de fluorita, durante o sopro e a avaliação da partição de fósforo com base em modelos de autores como Healy, Turkdogan, Basu e Suito, foi realizada utilizando os dados industriais da aciaria da ArcelorMittal Tubarão, com objetivo de comparar estes dados com as abordagens citadas. Um software termodinâmico foi usada para cálculo das atividades, da fração de líquido e do fósforo em equilíbrio, com a finalidade de auxiliar a identificação das principais diferenças termodinâmicas entre os dados experimentais e correntes. $\mathrm{O}$ resultado final mostra que as alterações realizadas no processo foram significativos para melhorar a taxa de partição de fósforo.

Palavras-chave: Desfosforação; Convertedor; Refino de aço.

\section{PHOSPHORUS DISTRIBUITION RATIO IN ARCELORMITTAL TUBARÃO BOF Abstract}

Phosphorus is one of the most important elements in the oxygen steelmaking production process, due to the difficulty of removing this element from the steel during the oxygen blowing process and mainly after taping from BOF to the steel ladle. This paper shows the results obtained by modifying some steel production variables, such as lime consumption, $\mathrm{FeO}$ in the slag and use of fluorspar during the blow, and comparing with current steel grades produced without any changes. Also, one evaluation of phosphorus partition models from authors such as Healy, Turkdogan, Basu and Suito, was made by using industrial data from ArcelorMittal Tubarão BOF in order to verify the approach of the phosphorus obtained to the equilibrium. To support the analyzes, a thermodynamic software was used to calculate activities, liquid fraction and phosphorus in equilibrium, with the purpose to indentify the main thermodynamic difference of current heats comparing with modified ones. The final result shows that the changes made in the process were significant to enhance the phosphorus partition rate.

Keywords: Dephosphorization; Converter; Steelmaking.

1 Engenheiro Metalurgista. Gerente de Produção de Aço, ArcelorMittal Tubarão, Vitória, ES, Brasil.

2 Engenheiro Metalurgista, Dr., Especialista de Processo de Produção de Aço, ArcelorMittal Tubarão, Vitória, ES, Brasil.

3 Engenheiro Químico, Mestrando em Engenharia Metalúrgica, Programa de Pós-Graduação em Engenharia Metalúrgica e de Materiais (PROPEMM), IFES, Vitória, ES, Brasil.

4 Engenheiro Metalurgista, Prof. Dr., graduação e pós-graduação em Engenharia Metalúrgica e de Materiais, Instituto Federal do Espírito Santo (IFES), Vitória, ES, Brasil.

\footnotetext{
* Contribuição técnica ao 450 Seminário de Aciaria - Internacional, 25 a 28 de maio de 2014,
} 


\section{INTRODUÇÃO}

Um equacionamento geral da desfosforação do aço, considerando que esta ocorra na interface metal-escória com a formação de $3 \mathrm{CaO} . \mathrm{P}_{2} \mathrm{O}_{5}$, que é o composto responsável pelo abaixamento da atividade do $\mathrm{P}_{2} \mathrm{O}_{5}$, na escória, e tomando os estados padrões, pode ser definido pela seguinte reação 1 [1].

$\underline{2 \mathrm{P}}+5(\mathrm{FeO})+3(\mathrm{CaO})=\left(3 \mathrm{CaO} \cdot \mathrm{P}_{2} \mathrm{O}_{5}\right)+5 \underline{\mathrm{Fe}}$

$\Delta G^{0}=-204.450+83,55 . T(\mathrm{~J} / \mathrm{mol})$

No Equacionamento 3 pode-se verificar os principais fatores termodinâmicos que influenciam a desfosforação: atividade do pentóxido de fósforo $\left(P_{2} \mathrm{O}_{5}\right)$ na escória líquida, temperatura, potencial de oxigênio na interface de reação, atividade do $\mathrm{CaO}$ da escória e coeficiente de atividade do fósforo no metal [2].

$$
\ln [\% P]=\frac{1}{2} \ln a_{\left(3 \mathrm{CaO} . \mathrm{P}_{2} \mathrm{O}_{5}\right)}+\frac{\Delta G^{0}}{2 R T}-\frac{5 \mu_{\mathrm{O}_{2}}}{4 R T}-\frac{3}{2} \ln a_{(\mathrm{CaO})}-\ln f_{P}
$$

Autores [1,3-5], estudaram o processo de partição de fósforo entre o aço e a escória conforme relação 4 , utilizando o coeficiente de partição de fósforo $\left(L_{P}\right)$ :

$$
L_{P}=\frac{(\% P)}{[\% P]}
$$

Onde $[\% \mathrm{P}]=$ teor de fósforo no metal e $(\% \mathrm{P})$ = teor de fósforo na escória.

Suito e Inoue [4] contribuindo com a abordagem de Balajiva et al. [3],acrescentou outros óxidos que afetam a partição do fósforo na formulação que já possui variáveis como teor de $\mathrm{CaO}$, \%FeT (Ferro total) e temperatura para escórias com concentração de $\mathrm{MnO}$ entre 2 e 5\%, obtendo a expressão mostrada na Equação 5:

$$
\begin{aligned}
& \log \left(L_{P}\right)=0,0720\left[(\% \mathrm{CaO})+0,3(\% \mathrm{MgO})+0,6\left(\% \mathrm{P}_{2} \mathrm{O}_{5}\right)+0,6(\% \mathrm{MnO})\right]+2,5 \log \left(\% \mathrm{Fe}_{t}\right)+ \\
& +\frac{11570}{T}-10,52
\end{aligned}
$$

Dentre estudos propostos por Healy [5], este trabalho utiliza a abordagem da Equação 6, para o cálculo do coeficiente de partição do fósforo:

$$
\log \left(L_{P}\right)=0,08 \cdot(\% C a O)+2,5 \log \left(\% F e_{\text {total }}\right)+\frac{22350}{T}-16
$$

Outra correlação de partição de fósforo, esta desenvolvida por Turkdogan [1], é mostrada na Equação 7 utilizando além das variáveis já citadas anteriormente, o oxigênio dissolvido no banho.

$$
\log \left(L_{P}\right)=\frac{21740}{T}-9,87+0,071 \cdot B O+2,5 \log [\% O]
$$

BO é definido como óxidos básicos, que fazem parte da escória, como é mostrado na Equação 8 e [\%O] a concentração de oxigênio dissolvido no banho, Equação 9.

$$
\begin{aligned}
& \mathrm{BO}=(\% \mathrm{CaO})+(\% \mathrm{CaF})_{2}+0,3(\% \mathrm{MgO}) \\
& {[\% \mathrm{O}]=(0,1 \cdot \mathrm{T}-155,3) \cdot 10^{-4} \cdot(\% \mathrm{FeO})}
\end{aligned}
$$

Basu et al. [6] propôs uma relação da partição de fósforo com a fração de cátions e de ânions da escória, conforme Equação 10.

* Contribuição técnica ao $45^{\circ}$ Seminário de Aciaria - Internacional, 25 a 28 de maio de 2014, 


$$
\log \left(L_{P}\right)=1,97 \cdot X_{\mathrm{Ca}^{2+}}+2,0 . X_{\mathrm{Fe}^{2+}}-2,04 \cdot X_{\mathrm{SiO}_{4}^{+4}}+\frac{6713}{T}-1,84
$$

Basu et al. [6] em seu trabalho mostra que a variação real da partição de fósforo durante o sopro de oxigênio varia entre 40 a $60 \%$ da aproximação do valor de equilíbrio.

Este trabalho relaciona as abordagens de partição de fósforo [1-5], com os dados reais de processo da ArcelorMittal Tubarão, tais como: adição de fundentes no convertedor (ex.: cal calcítica, cal dolomítica e fluorita), composição química da escória de fim de sopro e resultados obtidos entre as corridas com alteração (experimentais) e sem alteração (normais).

\section{MATERIAIS E MÉTODOS}

Os experimentos industriais foram realizados em modelos existentes no processo de fabricação de aço dos convertedores da ArcelorMittal Tubarão. Com base nos modelos mencionados, foram realizadas alterações em variáveis do processo com o objetivo de obtenção de teores de fósforo mais baixos que os realizados nas corridas normais (menores que 100 ppm).

Para este desenvolvimento, foram coletadas informações de 14 corridas produzidas sem nenhuma alteração do processo, que no estudo são chamadas de corridas normais, e 14 outras corridas com alteração nas variáveis de processo denominadas experimentais.

Todas as modificações realizadas no processo tiveram como objetivo um aumento da partição de fósforo. Uma destas variáveis é o teor de Ferro total na escória, ou teor de FeO na escória, como pode ser notado nas abordagens (5), (6), (7) e (10), todas estas mostram que um aumento do teor de Ferro na escória favorece a partição de fósforo [1-5]. Desta forma alterações com o objetivo de aumentar a oxidação da escória foram realizadas.

Outra variável que aparece como efeito positivo na partição de fósforo é o teor de $\mathrm{CaO}$ na escória, que pode ser verificado nos equacionamentos 5 a 8 e 10. A principal fonte de $\mathrm{CaO}$ no processo de produção de aço em convertedores a oxigênio são as Cales (calcitica e dolomitica).

A fluorita $\left(\mathrm{CaF}_{2}\right)$, fundente usado para melhorar a fluidez da escória, também é citada no Equacionamento 7, onde o incremento desta eleva a partição de fósforo.

A temperatura, apesar de não fazer parte das alterações de deste trabalho, tem papel determinante no processo de desfosforação, esta aparece em todos os modelos destacados anteriormente (1) a (10), porém como a temperatura tem que ser mantida elevada para absorver a perda térmica oriunda das adições de ligas de Mn para o aço produzido neste experimento (acalmado ao Alumínio e Silício), com Manganês em faixa, esta aparece apenas nos resultados como variação do processo.

A Tabela 1 mostra o resumo das ações nas variáveis do processo e o potencial de impacto destas no coeficiente de partição de fósforo, dos autores citados [1-6].

* Contribuição técnica ao $45^{\circ}$ Seminário de Aciaria - Internacional, 25 a 28 de maio de 2014, 
Tabela 1. Resumo das ações no processo e impacto na partição de fósforo ( $\left.L_{P}\right)$

\begin{tabular}{ccccc}
\hline Parâmetros & Ações & Quantidade & Medição & $L_{P}$ \\
\hline CaO equivalente $^{*}$ & Aumentar Cal & $+3 \mathrm{~kg} / \mathrm{t}$ aço & Peso de Material & $\uparrow$ \\
Fluorita & Aumentar & $+1 \mathrm{~kg} / \mathrm{t}$ aço & Peso de Material & $\uparrow$ \\
& ${\text { Elevar } \mathrm{VO}_{2}{ }^{* *}}^{\text {Oxidação }}$ & $+3 \mathrm{Nm} 3 / \mathrm{t}$ aço & $\%$ FeO e FeT na & $\uparrow$ \\
& Elevar lança $\mathrm{O}_{2}$ & $200 \sim 400 \mathrm{~mm}$ & escória & $\uparrow$ \\
Temperatura & Manter & - & Fim de sopro $\left({ }^{\circ} \mathrm{C}\right)$ & $\rightarrow$ \\
\hline
\end{tabular}

A composição química do gusa utilizado neste trabalho pode ser verificado na Tabela 2.

Tabela 2. Valores de composição média do gusa nas corridas normais e experimentais

\begin{tabular}{ccccccc}
\hline & \multicolumn{5}{c}{ Composição Química do Gusa (\%) } & T emp \\
\cline { 2 - 6 } & C & Si & P & Mn & S & (oC) \\
\hline Media Exp & 4,74 & 0,24 & 0,067 & 0,43 & 0,002 & 1366 \\
\hline Média Normais & 4,49 & 0,14 & 0,083 & 0,52 & 0,003 & 1380 \\
\hline Nota: Media Exp - Média das corridas experimentais. & & & &
\end{tabular}

A coleta das amostras de gusa foram realizadas na estação de dessulfuração (KR) antes do carregamento nos convertedores, e as amostras de aço através de amostradores acoplados a sublança.

A análise químicas foram realizadas no laboratório da ArcelorMittal Tubarão.

Para auxiliar a avaliação dos resultados obtidos, a luz da termodinâmica, foi utilizado software de Termodinâmica computacional, FactSage 6.2.

\section{RESULTADOS E DISCUSSÃO}

Os resultados obtidos nos experimentos são comparados a seguir com as corridas normais para avaliação da eficácia das alterações no processo de desfosforação e de partição de fósforo.

$\mathrm{Na}$ Tabela 3 têm-se os valores de composição química média obtida das corridas normais e experimentais

Tabela 3. Valores médios de fim de sopro das corridas normais e experimentais

\begin{tabular}{lccccccc}
\hline \multicolumn{7}{c}{ Composição Química do Aço (\%) } & \multirow{2}{*}{ T ( $\left.{ }^{\circ} \mathbf{C}\right)$} \\
\hline Exp & Fe & C & Si & Mn & P & S & \\
Normais & 99,857 & 0,038 & 0,001 & 0,092 & 0,009 & 0,004 & 1699 \\
\hline
\end{tabular}

Pode-se notar que os valores de fósforo obtidos para as corridas experimentais foram significativamente mais baixos que os valores das corridas normais. Mostrando que as alterações no processo foram suficientes para obtenção de fósforos mais baixos.

$\mathrm{Na}$ Tabela 4, pode-se verificar os valores da composição química da escória das corridas experimentais e normais.

* Contribuição técnica ao 450 Seminário de Aciaria - Internacional, 25 a 28 de maio de 2014, Porto Alegre, RS, Brasil. 
Tabela 4. Valores de composição média da escória das corridas normais e experimentais

Composição Química da Escória (\%)

\begin{tabular}{lcccccccc}
\hline & FeO & CaO & SiO2 & MnO & P2O5 & Al2O3 & MgO & CaF2 \\
\cline { 2 - 9 } Exp & 33,03 & 42,06 & 10,28 & 2,91 & 1,42 & 1,44 & 7,36 & 1,50 \\
Normais & 31,31 & 40,95 & 10,61 & 4,94 & 1,71 & 1,93 & 8,11 & 0,44 \\
\hline
\end{tabular}

Pela análise química da escória, pode-se notar que o teores de $\mathrm{FeO}$ e $\mathrm{CaO}$ das corridas experimentais são maiores que os das corridas normais. Nota-se também que os valores de $\mathrm{CaF}_{2}$ são superiores para as corridas experimentais.

Pela análise da Equação 3, pode-se afirmar que as corridas experimentais deveriam apresentar menores teores de fósforo final, o que realmente ocorreu, como mostra o resultado da Tabela 3, onde a concentração média de fósforo no metal nas corridas normais é de 0,015\% e nas experimentais é de 0,009\%.

Através dos valores obtidos de análise química do aço e escória, foram calculados os valores de partição de fósforo baseados nas Equações 4-7 e 10, como pode ser visualizado na Tabela 5.

Tabela 5. Valores de $L_{p}$ real e calculados de acordo com os quatro modelos apresentados

\begin{tabular}{lccccc}
\hline & $L_{P(4) \text { Real }}$ & $L_{P(5)}$ & $L_{P(6)}$ & $L_{P(7)}$ & $L_{P(10)}$ \\
\hline Exp & 76,3 & 175,5 & 170,5 & 181,1 & 200,5 \\
Normais & 50,5 & 177,4 & 135,4 & 122,0 & 189,7 \\
\hline
\end{tabular}

Nota: $L_{P(4)}: L_{P}$ calculado através da Equação 4, com valores reais; $L_{P(5)}$ : $L_{P}$ calculado através da Equação 5 Modelo de Suito e Inoue [4] $L_{P(6)}$ : $L_{P}$ calculado através da Equação 6 - Modelo de Healy [5]; $L_{P(7)}: L_{P}$ calculado através da Equação 7 - Modelo de Turkdogan [1]; $L_{P(10)}$ : $L_{P}$ calculado através da Equação 10 - Modelo de Basu [6].

Os valores de $L_{p}$ encontrados para os diferentes modelos descritos no presente trabalho mostrados na Tabela 5 , mostram-se coerentes com os encontrados na literatura [1-6].

Analisando a Tabela 5, nota-se que houve um aumento nos coeficientes de partição de fósforo para as corridas experimentais em 3 dos 4 modelos apresentados.

A relação definida por Basu [6], citada anteriormente, entre a partição de fósforo real (LPreal) e a partição dos modelos em equilíbrio é apresentadas na Figura 1.

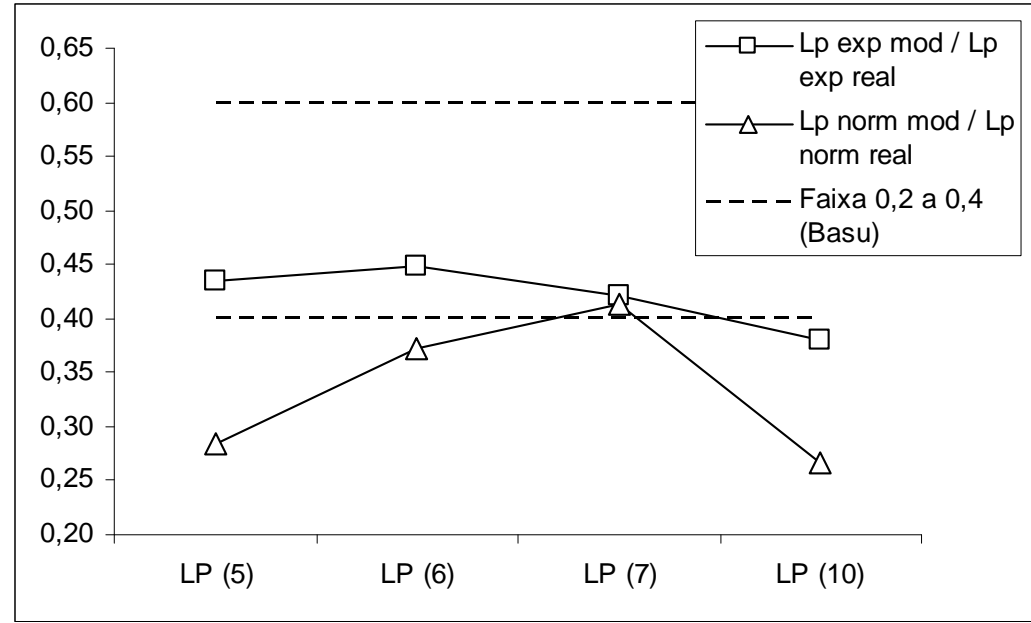

Figura 1. Relações entre a partição real $\left(L_{P(\text { real })}\right)$ e a partição em equilíbrio.

Pela análise da Figura 1 nota-se que a relação entre a partição real $\left(L_{\text {Preal }}\right)$ e a partição no equilíbrio, no presente trabalho, está entre 0,27 a 0,45 . Somente a

* Contribuição técnica ao 45 Seminário de Aciaria - Internacional, 25 a 28 de maio de 2014, 
partição encontrada usando a fórmula de Turkdogan [1] ( $\left.L_{\text {Preal }} / L_{P}{ }_{(7)}\right)$ apresentou valores dentro da aproximação do equilíbrio citado por Basu et al. [6].

Utilizando o modelo de Turkdogan [1] como referencia, foi avaliada a influência da parcela BO, Equação 8, no coeficiente de partição de fósforo, que pode ser verificado na Figura 2.

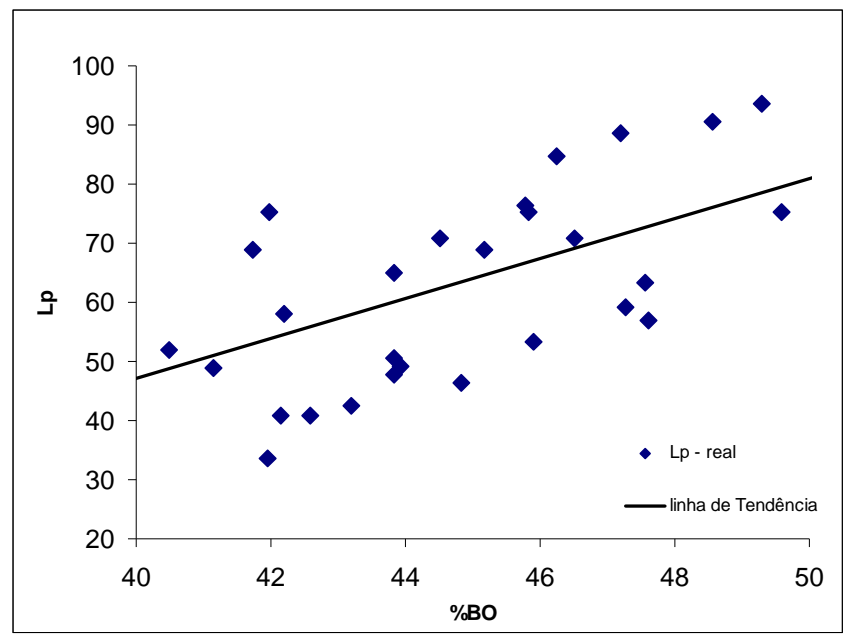

Figura 2. Variação do coeficiente de partição de fósforo real comparados com $B O=(\% \mathrm{CaO})+0.3(\% \mathrm{MgO})+\left(\% \mathrm{CaF}_{2}\right)$.

Pode-se verificar na Figura 2, uma correlação do coeficiente de partição de fósforo com o valor de $\mathrm{BO}$, Equação 8, mostrando que as variáveis contidas em $\mathrm{BO}(\mathrm{CaO}$, $\mathrm{MgO}$ e $\mathrm{CaF}_{2}$ ) tem influência significativa na partição de fósforo. No extremo direito do gráfico nota-se os maiores valores de partição de fósforo, podendo citar que a melhor composição para BO para este trabalho é de 46\% a 50\%.

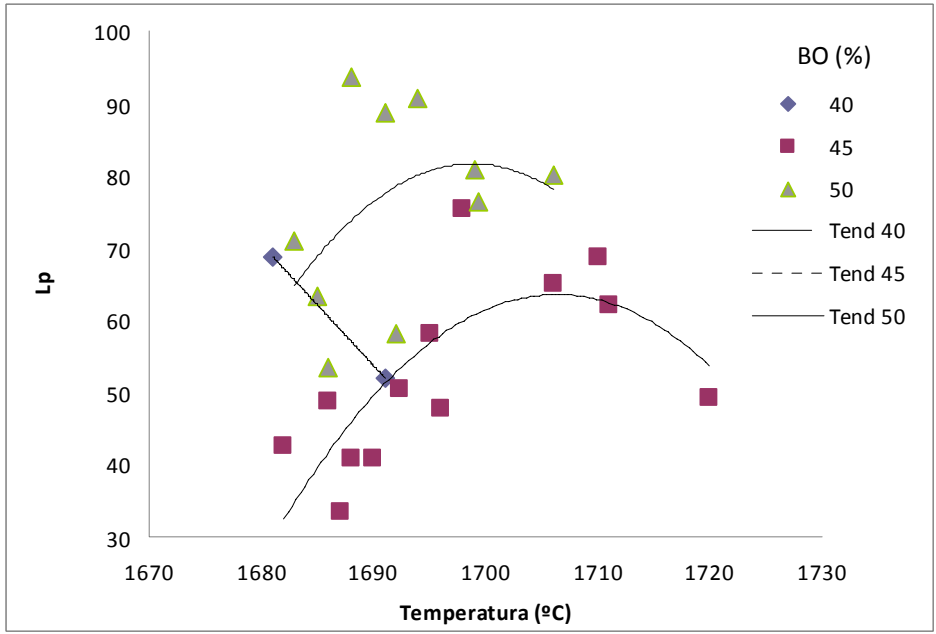

Figura 3. Influência da Temperatura em BO. $\mathrm{BO}=(\% \mathrm{CaO})+0.3(\% \mathrm{MgO})+\left(\% \mathrm{CaF}_{2}\right)$.

Nota-se na Figura 3, que o aumento de BO gera uma um acréscimo em $L_{p}$, porém o efeito da elevação de temperatura impacta significativamente no efeito positivo de $\mathrm{BO}$, de forma a reduzir a influência deste em temperatura mais elevadas. Este gráfico demonstra que para temperaturas mais elevadas, BO não é tão significativo.

* Contribuição técnica ao $45^{\circ}$ Seminário de Aciaria - Internacional, 25 a 28 de maio de 2014 , Porto Alegre, RS, Brasil. 
Ampliando o foco na Equação 8, três são as variáveis que participam desta, teor de $\mathrm{CaO}, \mathrm{MgO}$ e $\mathrm{CaF}_{2}$ na escória. A seguir é destacada a participação de cada uma e seu efeito no coeficiente de partição de fósforo.

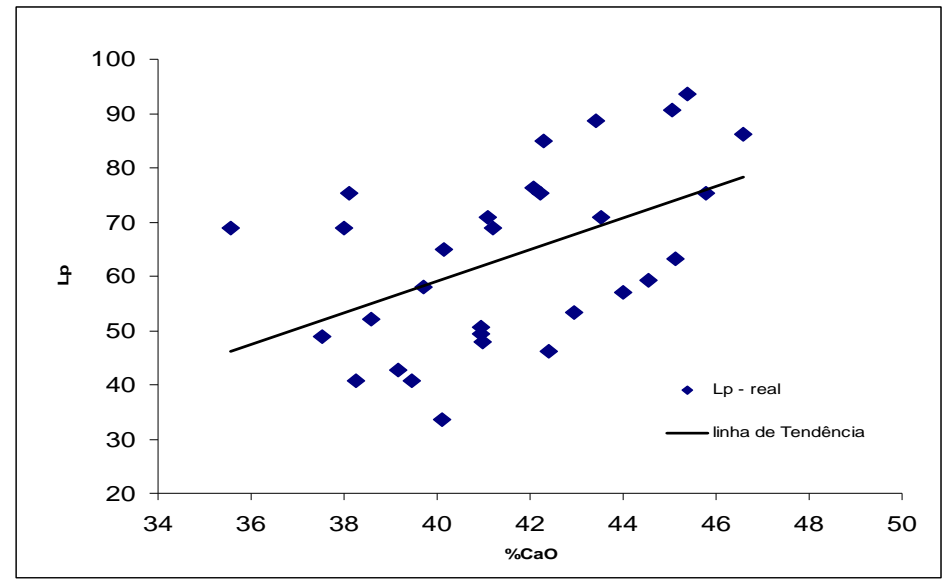

Figura 4. Efeito do Teor de CaO na escória no coeficiente de partição de fósforo $\left(L_{P}\right)$.

Na Figura 4, nota-se que o incremento do teor de CaO na escória, gera um aumento no coeficiente de partição de fósforo, apesar da grande dispersão, pois existem corridas com teor de $\mathrm{CaO}$ baixos (menores que 39\%) que apresentaram resultados de $L_{P}$ superiores a 65 . Isto mostra que não só o teor de $\mathrm{CaO}$ na escória foi determinante para a melhorar o processo de desfosforação.

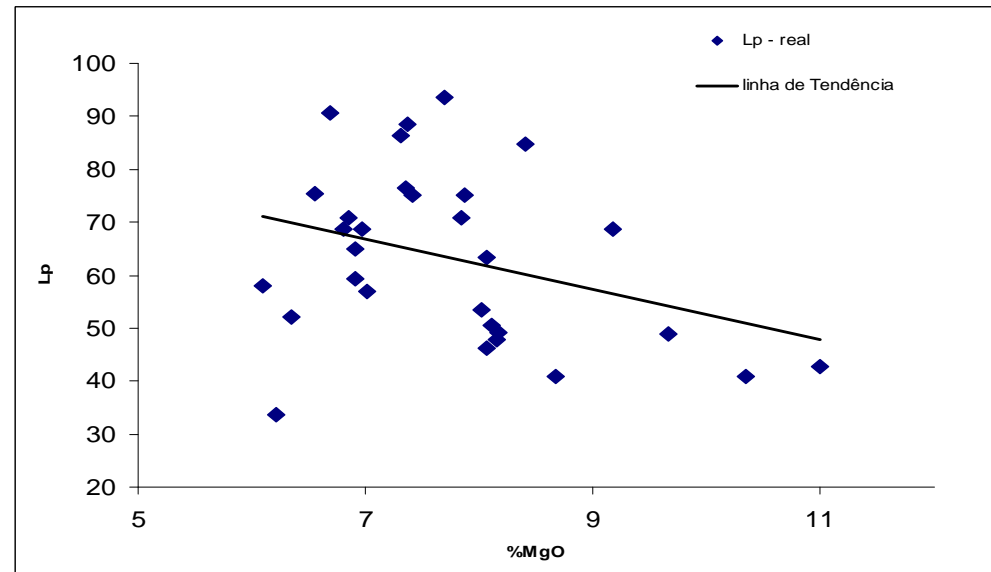

Figura 5. Efeito do Teor de MgO no coeficiente de partição de fósforo $\left(L_{P}\right)$.

Os resultados da Figura 5 mostram que o aumento de MgO gera uma redução do coeficiente de partição de fósforo. Resultado que está em desacordo com a abordagem de Turkdogan [1], que considera o efeito do MgO equivalente a 0,3 do peso do $\mathrm{CaO}$ para a Equação 8.

B. Deo e co-autores [7] cita em seu trabalho que valores elevados de $\mathrm{MgO}$, da ordem de $7 \%$ na escória, impactam negativamente no coeficiente de partição de fósforo. 

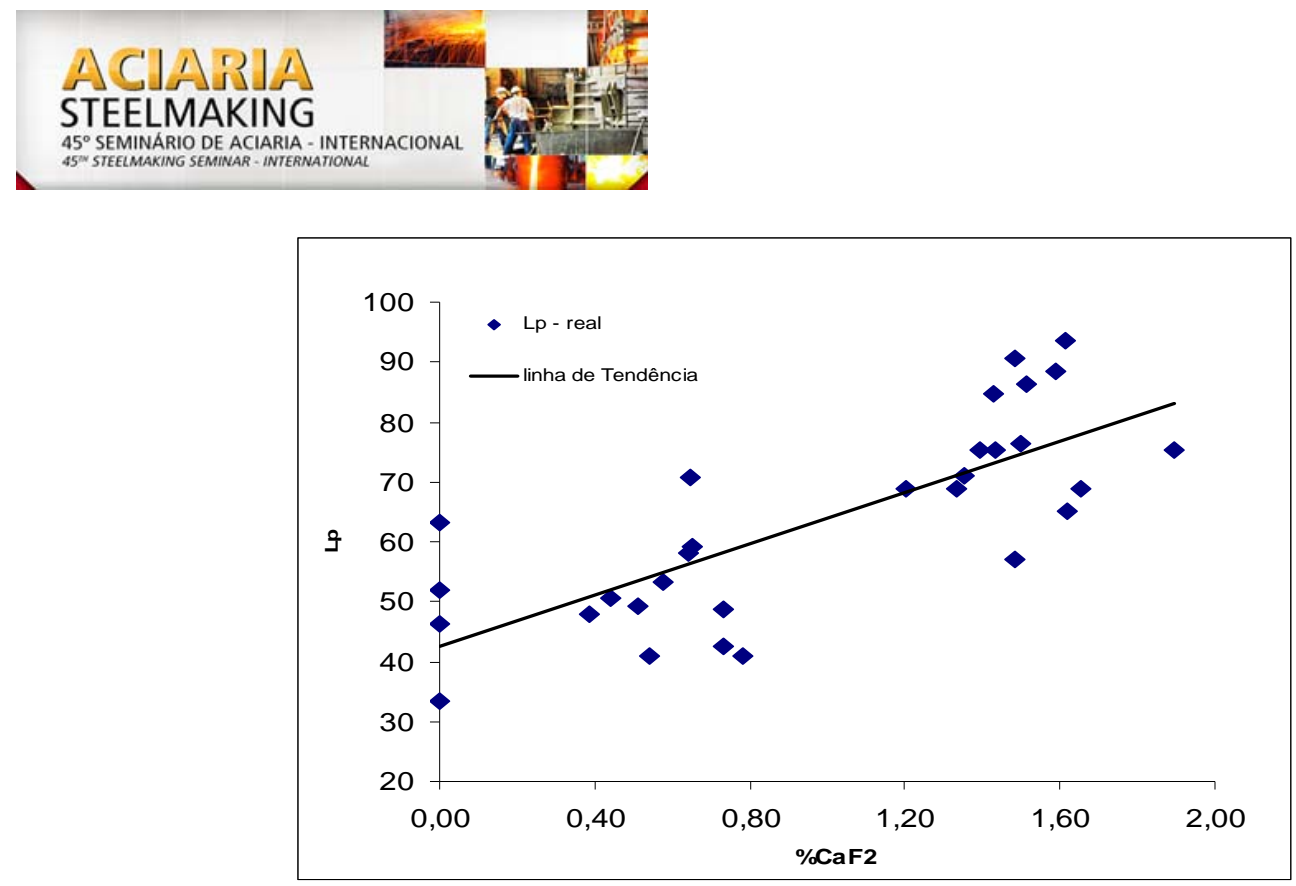

Figura 6. Efeito do Teor de $\mathrm{CaF}_{2}$ no coeficiente de partição de fósforo $\left(L_{P}\right)$.

Nota-se pela Figura 6 , uma a grande influência do $\mathrm{CaF}_{2}$ no coeficiente de partição de fósforo, principalmente para valores entre 1,20 a 1,80\% de $\mathrm{CaF}_{2}$ na escória. Esta influência pode ser relacionada ao estudo realizado por Suito e Inoue [8] que demonstra que a elevação de $\mathrm{CaF}_{2}$ na escória aumenta o valor da constante de equilíbrio $\left(K_{P}\right)$ além de baixar o coeficiente de atividade do $\mathrm{P}_{2} \mathrm{O}_{5}$, que favorece a desfosforação.

Os resultados mostram que os valores baseados nos modelo de Turkdogan [1], o qual usa o valor de BO como referencia, teve influência positiva para os teores de $\mathrm{CaO}$ e $\mathrm{CaF}_{2}$ e negativo para o teor de $\mathrm{MgO}$ no processo de desfosforação do aço. Para auxiliar avaliação dos resultados de partição de fósforo, foi utilizada a termodinâmica computacional, com determinação de dados em condições de equilíbrio. Os dados de saída desta simulação são mostrados na Tabela 6 .

Tabela 6. Valores médios obtidos na termodinâmica computacional

\begin{tabular}{lccccccc}
\hline \multicolumn{8}{c}{ Equilíbrio } \\
\hline & \%Peq & $\begin{array}{c}\text { \%Lí- } \\
\text { quido }\end{array}$ & $\begin{array}{c}\text { \%Sóli- } \\
\text { do }\end{array}$ & a (CaO) & a (FeO) & a (3CaO.P2O5) & $\begin{array}{c}\text { Visco- } \\
\text { sidade }\end{array}$ \\
\hline Exp & 0,0020 & 97,3 & 2,7 & 0,64 & 0,24 & 0,016 & 0,271 \\
Normais & 0,0030 & 96,9 & 3,1 & 0,61 & 0,24 & 0,033 & 0,283 \\
\hline
\end{tabular}

Nota: \%Peq - Teor de fósforo em equilíbrio; a(CaO) - Atividade do $\mathrm{CaO}$; $\mathrm{a}\left(3 \mathrm{CaO}_{2} \mathrm{P}_{2} \mathrm{O}_{5}\right)$ - Atividade do $3 \mathrm{CaO} \cdot \mathrm{P}_{2} \mathrm{O}_{5}$.

Com os resultados obtidos a Tabela 6, pode-se avaliar a influência das alterações no processo de desfosforação. Os valores mais baixos de fósforo em equilíbrio, atividade do $3 \mathrm{CaO} . \mathrm{P}_{2} \mathrm{O}_{5}$ e viscosidade para as corridas experimentais, como também os valores mais elevado da atividade do $\mathrm{CaO}$ e percentual de liquido, para estas mesmas corridas, corroboram para obtenção de coeficientes de partição de fósforos mais elevados do que os gerados para corridas normais. Nota-se também que não houve alteração no valor da atividade do FeO.

Este trabalho mostra que as alterações realizadas no processo foram suficientes para obtenção dos resultados de fósforo no fim de sopro mais baixos, onde as corridas experimentais foram em média $0,006 \%$ menores que os das corridas normais.

* Contribuição técnica ao 45 Seminário de Aciaria - Internacional, 25 a 28 de maio de 2014, Porto Alegre, RS, Brasil. 


\section{CONCLUSÕES}

Comparando as alterações realizadas nas varáveis de processo com as análises dos resultados obtidos de partição de fósforo e dos valores termodinâmicos, as seguintes conclusões podem ser observadas:

- Dentre os modelos de partição de fósforo estudados, o que melhor representou os dados retratados neste trabalho foi o modelo de Turkdogan [1];

- Os valores de BO, Equação 8, mostraram uma correlação significativa com os resultados de partição de fósforo, onde os melhores valores se encontram entre 46 a $50 \%$ de $\mathrm{BO}$;

- O teor de $\mathrm{CaO}$ na escória apresentou uma correlação significativa com o coeficiente de partição de fósforo, com resultados mais significativos entre a 43 a $46 \% \mathrm{CaO}$;

- O teor de $\mathrm{CaF}_{2}$ na escória influenciou significativamente o coeficiente de partição de fósforo, com os melhores resultados na faixa entre 1,4 a 1,8\% de $\mathrm{CaF}_{2}$ na escória;

- O aumento do teor de MgO na escória tem impacto negativo no coeficiente de partição de fósforo, onde os melhores resultados obtidos foram para valores inferiores a $8 \%$ na escória.

Os resultados de fósforo obtidos nas corridas experimentais foram significativamente superiores às normais, mostrando que as alterações realizadas no processo foram eficazes para atingir os resultados esperados.

\section{Agradecimentos}

Os autores agradecem a todos que participaram do processo de desenvolvimento da produção dos aços baixo fósforo na área de refino, bem como o suporte do laboratório de análises químicas da aciaria da ArcelorMittal Tubarão.

\section{REFERÊNCIAS}

1 Turkdogan ET. Fundamentals of Steelmaking. $1^{\mathrm{a}}$ ed. London: The Institute of Materials, 1996. p.331.

2 Campos VF. Tecnologia de Fabricação do Aço Líquido. $3^{\mathrm{a}}$ ed. Belo Horizonte: Universidade Federal de Minas Gerais; 1985. p.259.

3 Balajiva K, Quarrel AG, Vajragupta P. Journal of the Iron and Steel Institute. 1946;153:115. Apud: Turkdogan ET. Assessment of $\mathrm{P}_{2} \mathrm{O}_{5}$ activity in Molten Slags. ISIJ International. 2000;40(10): 964-70.

4 Suito $\mathrm{H}$, Inoue R. Thermodynamic assessment of hot metal and steel dephosphorisation with MnO-containing BOF slags. ISIJ International. 1995;35(3):258-265.

5 Healy GW. A new look at phosphorus distribution. Journal of Iron and Steel Institute; 1971;208:664-68.

6 Basu S, Lahiri AK, Seetharaman S. A model for activity coefficient of P2O5 in BOF slag and phosphorus distribution between liquid steel and slag. ISIJ International. 2007;47(8):1236-38.

7 Deo B, Halder J, Snoeijer B, Overbosch A, Boom R. Effect of MgO and Al2O3 variations in oxygen steelmaking (BOF) slag on slag morphology and phosphorus distribution. Ironmaking and Steelmaking. 2005;32(1):503-08.

8 Suito H, Inoue R. Effect of Calcium Fluoride on Phosphorus Distribution between MgO Saturated Slags of the System CaO-MgO-FeO-SiO2. ISIJ International. 2006;46(2):17479.

* Contribuição técnica ao $45^{\circ}$ Seminário de Aciaria - Internacional, 25 a 28 de maio de 2014, 